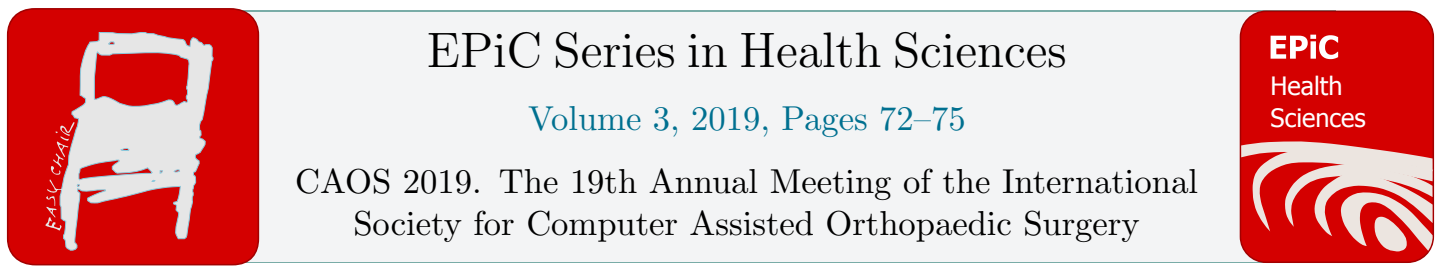

\title{
Healthcare Utilization and Payer Cost Analysis of Robotic-Arm Assisted Total Knee Arthroplasty at 30-, 60-, and 90-Days
}

\author{
Andrea Coppolecchia, MPH1; Christina Cool, MPH2; David Jacofsky, MD3; David \\ Gregory, MPA, FACHE2; Nipun Sodhi, MD4; Michael A. Mont, MD4 \\ ${ }^{1}$ Stryker Corporation, Mahwah, NJ \\ ${ }^{2}$ Bakey Tilly, Chicago, IL \\ ${ }^{3}$ The Core Institute, Pheonix, AZ \\ ${ }^{4}$ Lenox Hill Hospital, Northwell Health, New York, New York
}

\begin{abstract}
Introduction

This study performed a healthcare utilization analysis between robotic-arm assisted and manual TKA techniques at three intervals to better understand intra-episode trends that can lead to optimized care pathways. Specifically, we compared: (1) index costs; and (2) discharge dispositions; as well as (3) 30-; (4) 60-; and (5) 90day: a) total episode-of-care costs, b) post-operative healthcare utilization, and c) readmissions.
\end{abstract}

\section{Methods}

The Medicare 100\% Standard Analytical Files was queried for robotic and manual TKAs (rTKA and mTKA) performed between January 1, 2016 and March 31, 2017. Based on strict inclusion and exclusion criteria, and 1:5 propensity score matching, 519 robotic and 2,595 manual TKA patients were analyzed. Total episode payments, healthcare utilization, and readmissions, at 30-, 60-, and 90-day time points were compared between cohorts with a generalized Linear Model, Binomial Regression, log link, Mann-Whitney, and Pearson's Chi Squared tests with $\mathrm{p}<0.05$ for statistical significance. 


\section{Results}

The robotic vs. manual cohort average total episode payment was $\$ 17,768$ vs. $\$ 19,899$ ( $\mathrm{p}<0.0001)$ at 30 -days; $\$ 18,174$ vs. $\$ 20,492(\mathrm{p}<0.0001)$ at 60 -days; and $\$ 18,568$ vs. $\$ 20,960$ ( $\mathrm{p}<0.0001)$ at 90 -days. At 30 days, $47 \%$ fewer rTKA patients utilized SNF services (13.5 vs. $25.4 \%, \mathrm{p}<0.0001$ and had lower SNF costs at 30( $\$ 6,416$ vs. $\$ 7,732 ; p=0.0040), 60$ - (\$6,678 vs. $\$ 7,901, p=0.0072)$, and 90-days $(\$ 7,201$ vs. $\$ 7,947, p=0.0230)$. rTKA patients also utilized fewer home-health visits and costs at each time point $(\mathrm{p}<0.05)$. Additionally, 31.3\% fewer rTKA patients utilized emergency room services at 30-days postoperatively. The robotic cohort had significantly fewer 90-day readmissions (5.20 vs. 7.75\%; $\mathrm{p}=0.0423$ ).

\section{Discussion}

Robotic TKA is associated with significantly lower 30-, 60-, and 90-day postoperative costs and healthcare utilization. These results are of marked importance given the emphasis to contain and reduce healthcare costs for total joints arthroplasties. This analysis provides initial economic insight into robotic-arm assisted TKA with promising results.

\section{Manuscript}

\section{Introduction}

Robotic technology has been shown to have the potential to help improve clinical, radiographic, and patient-reported outcomes after total knee arthroplasty (TKA) ${ }^{1}$. This interactive system allows for real-time intra-operative assessments, virtually evaluating ranges-of-motion, ligament tension, and overall knee alignment ${ }^{2}$. The haptically-bounded saw blade provides immediate, pulsatile feedback while making bone cuts ${ }^{3,4}$, which helps reduce the chances of bony or soft-tissue cuts outside of the desired window. Combined, these advantages can help orthopaedic surgeons optimize TKA surgery, even in difficult cases, to help improve patient outcomes, and to potentially improve operating room efficiencies ${ }^{2,5-7}$. Therefore, the purpose of this study was to perform a healthcare utilization analysis between robotic-arm assisted TKA and manual TKA techniques. Specifically, we compared: (1) index costs and (2) discharge dispositions; as well as (3) 30-; (4) 60-; and (5) 90-day: a) total episode-of-care (EOC) costs, b) post-operative healthcare utilization, and c) readmissions. 


\section{Methods}

The Medicare 100\% Standard Analytical Files was queried for robotic and manual TKAs (rTKA and mTKA) performed between January 1, 2016 and March 31, 2017. After strict inclusion and exclusion criteria, and 1:5 propensity score matching based on age, sex, race (as defined by CMS), geographic division (based on U.S. Census Bureau divisions), and high-cost comorbidities (which include: Hypertension, Diabetes, Coronary Artery Disease (CAD), Chronic Obstructive Pulmonary Disease (COPD), Smoking, Pneumonia, Cancer, and Obesity), 519 robotic and 2,595 manual TKA patients were analyzed. Total episode payments, healthcare utilization, and readmissions, at 30-, 60-, and 90-day time points were compared between cohorts with a generalized Linear Model, Binomial Regression, log link, Mann-Whitney, and Pearson's Chi Squared tests with $\mathrm{p}<0.05$ for statistical significance.

\section{Results}

The robotic vs. manual cohort average total episode payment was $\$ 17,768$ vs. $\$ 19,899(\mathrm{p}<0.0001)$ at 30 -days; $\$ 18,174$ vs. $\$ 20,492(\mathrm{p}<0.0001)$ at 60 -days; and $\$ 18,568$ vs. $\$ 20,960(\mathrm{p}<0.0001)$ at 90 -days. At 30 days, $47 \%$ fewer rTKA patients utilized SNF services (13.5 vs. 25.4\%, $<<0.0001$ and had lower SNF costs at 30( $\$ 6,416$ vs. $\$ 7,732 ; p=0.0040), 60$ - (\$6,678 vs. $\$ 7,901, p=0.0072)$, and 90-days $(\$ 7,201$ vs. $\$ 7,947, \mathrm{p}=0.0230)$. rTKA patients also utilized fewer home-health visits and costs at each time point $(\mathrm{p}<0.05)$. Additionally, 31.3\% fewer rTKA patients utilized emergency room services at 30-days postoperatively. The robotic cohort had significantly fewer 90-day readmissions (5.20 vs. 7.75\%; $\mathrm{p}=0.0423$ ).

\section{Discussion}

The continuous rise in healthcare costs has placed substantial economic burdens on providers, facilities, payers and patients. Therefore, a number of strategies have been developed in attempts to maintain or even decrease these costs. One avenue of pursuit is the introduction of technology in the operating room that is intended to drive efficiencies, decrease cost, and improve outcomes. The results from this study show that robotic TKA is associated with significantly lower 30-, 60-, and 90-day post-operative EOC costs. In addition, 90-day readmissions were significantly lowered. These lower costs can likely be associated with shorter lengths-of-stay, higher rates of discharge to home, reduced post-operative resource utilization, and fewer readmissions. Based on this data, robotic-arm assisted surgery appears to be a cost-effective option, and potentially provides added value for patients undergoing TKA. These findings may especially inform those in value-based contracting settings. 


\section{References}

1. Khlopas A, Sodhi N, Sultan AA, Chughtai M, Molloy RM, Mont MA. Robotic Arm-Assisted Total Knee Arthroplasty. J Arthroplasty United States, 2018;

2. Marchand RC, Sodhi N, Bhowmik-Stoker M, Scholl L, Condrey C, Khlopas A, et al. Does the Robotic Arm and Preoperative CT Planning Help with 3D Intraoperative Total Knee Arthroplasty Planning? J Knee Surg Germany, 2018;

3. Khlopas A, Chughtai M, Hampp EL, Scholl LY, Prieto M, Chang T-C, et al. Robotic-Arm Assisted Total Knee Arthroplasty Demonstrated Soft Tissue Protection. Surg Technol Int United States, 2017;30:441-446.

4. Hampp EL, Chughtai M, Scholl LY, Sodhi N, Bhowmik-Stoker M, Jacofsky DJ, et al. Robotic-Arm Assisted Total Knee Arthroplasty Demonstrated Greater Accuracy and Precision to Plan Compared with Manual Techniques. J Knee Surg Germany, 2018;

5. Sodhi N, Khlopas A, Piuzzi NS, Sultan AA, Marchand RC, Malkani AL, et al. Erratum to: The Learning Curve Associated with Robotic Total Knee Arthroplasty. J Knee Surg Germany, 2018;31(4):370.

6. Marchand RC, Sodhi N, Khlopas A, Sultan AA, Harwin SF, Malkani AL, et al. Patient Satisfaction Outcomes after Robotic Arm-Assisted Total Knee Arthroplasty: A Short-Term Evaluation. J Knee Surg 2017;

7. Kayani B, Konan S, Tahmassebi J, Pietrzak JRT, Haddad FS. Roboticarm assisted total knee arthroplasty is associated with improved early functional recovery and reduced time to hospital discharge compared with conventional jig-based total knee arthroplasty. Bone Joint J England, 2018;100-B(7):930-937. 